\title{
Effects of baffle reflection and shadow in an integrating sphere on the total luminous flux measurement of a linearly- shaped lamp
}

\author{
Kamol Wasapinyokul ${ }^{1,2}$, Santhad Chuwongin ${ }^{1,2}$, Ajchara Charoensook $^{2}$ \\ ${ }^{1}$ College of Advanced Manufacturing Innovation, King Mongkut's Institute of Technology Ladkrabang, 1, Soi Chalongkrung 1, Ladkrabang, \\ Bangkok, 10520, Thailand \\ ${ }^{2}$ National Institute of Metrology (Thailand), 3/4-5, Moo 3, Klong 5, Klong Luang, Pathumthani, 12120, Thailand
}

\begin{abstract}
The measured total luminous flux of a linearly-shaped lamp by using the integrating sphere substitution method against a standard spherical lamp can be deviated from accurate by the presence of a baffle in the sphere. The baffle introduced two main effects on the sphere response: the baffle reflection, or high-signal region, and the baffle shadow, or low-signal region. Once the baffle condition changed, the two effects changed, causing the measured value to change differently regarding the lamp alignment. In a perpendicular alignment, increasing the baffle length increased the measured flux value. This was due to the dramatic increase in the magnitude of the signal in the baffle reflection behind the baffle. In a coaxial alignment, on the contrary, increasing the baffle length resulted in the decrease of the measured flux value. This was due to the increase in the area of the baffle shadow on the opposite hemisphere from the baffle, which increased at a higher ratio than that of the baffle reflection. In both alignments, the measurement uncertainty increased with the baffle length due to the increasing magnitude of the signal fluctuations. The trends were similar for all linearlyshaped test lamps with different lengths and diameters.
\end{abstract}

\section{Section: RESEARCH PAPER}

Keywords: Integrating sphere; total luminous flux; linearly-shaped lamp; baffle; spatial correction factor

Citation: Kamol Wasapinyokul, Santhad Chuwongin, Ajchara Charoensook, Effects of baffle reflection and shadow in an integrating sphere on the total luminous flux measurement of a linearly-shaped lamp, Acta IMEKO, vol. 6, no. 4, article 17, December 2017, identifier: IMEKO-ACTA-06 (2017)-04-17

Section Editor: Marco Tarabini, Politecnico di Milano, Italy

Received February 1, 2017; In final form July 24, 2017; Published December 2017

Copyright: (C) 2017 IMEKO. This is an open-access article distributed under the terms of the Creative Commons Attribution 3.0 License, which permits unrestricted use, distribution, and reproduction in any medium, provided the original author and source are credited

Corresponding author: Kamol Wasapinyokul, e-mail: kamol.wa@kmitl.ac.th

\section{INTRODUCTION}

The integrating sphere substitution method is a method to realise the total luminous flux of a light source [1]-[5]. The method generally comprises an integrating sphere, a standard light source with a known total luminous flux value, and a test light source, the flux of which is to be measured. The integrating sphere is a hollow sphere, the inner wall of which is coated with a highly reflective material. Once there is a light source inside, due to the high reflective spherical-shaped wall, multiple reflections of the luminous flux occurs. This leads to the illuminance of the light due to the light source at every point on the sphere wall to be theoretically equal to each other. A photo-detector, installed somewhere on the sphere inner wall, then detects and measures this uniform illuminance in a form of an electronic signal. In order to measure the total luminous flux of the test source, both the standard and test sources would be, one at a time, installed at the centre of the sphere. The electronic signals corresponding to the illuminance of each lamp, detected by the photo-detector, are consequently collected. As the total luminous flux of the standard source is known, such value for the test source can be calculated, hence the name substitution method.

Accuracy of the measured total luminous flux value is generally a topic of interest in the researches [6]-[9]. For the integrating sphere substitution method, a source of the inaccuracy is the spatial non-uniformity of the illuminance on the sphere wall [9], [10]. Such non-uniformity is partly attributed to the occurrence of a crucial component in the sphere called baffle. The responsibility of a baffle can be explained as follows: As the photo-detector is to detect the 
uniform illuminance due to multiple reflections of the flux from the sphere wall, it must not detect the direct flux from the light source. Thus a thin opaque plate called baffle is needed to be installed between the light source and the photo-detector; its duty is to shield the direct flux from the light source not to incident directly on the photo-detector. The baffle is a necessary component of an integrating sphere; however, it is alien to the plain sphere wall. The uniformity of illuminance is thus destroyed, leading to the inaccurately measured total luminous flux of the test source.

This inaccuracy in the measured value is enhanced when the luminous intensity distributions of the standard and test sources become increasingly different. An apparent example of such case is when the standard light source is a typical spherical incandescent lamp while the test light source is of linear shape, such as a linear fluorescent lamp or a linear LED lamp. Moreover, the alignment of a linearly-shaped lamp in an integrating sphere can be varied, leading to different baffle conditions, hence the different non-uniformity characteristics of the sphere response.

A number of methods have been reported to reduce the spatial non-uniformity of the sphere caused by the baffle. These include increasing the number of photo-detectors on the sphere wall [9], [10] and improving the response of the detector [11]. These methods, however, require either modification of the sphere or changes of the sphere components, both of which may not be economically or practically suitable for an existing sphere. Some reports studied the effects of baffle on the sphere uniformity. It has been reported that the baffle characteristics, such as its shape and reflectance, could increase and decrease the measured total luminous flux value through its reflection and shadow, respectively [7], [12], [13]. This method could be used to reduce the spatial non-uniformity of the sphere. However, the reports have studied only in the cases where the standard and test light sources were not much different in shape. There is still a lack of study on the effects of baffle in an integrating sphere on the measurement of the total luminous flux of a linearly-shaped lamp by using a typical spherical standard lamp, even though such measurements have been practiced generally due to the extensive use worldwide of the linearly-shaped lamps.

This paper reports the effects of baffle in an integrating sphere on the total luminous flux measurement of a linearlyshaped lamp at two different lamp alignments: perpendicular and coaxial alignments. Two baffle effects - baffle reflection and baffle shadow - have been varied; their concurrent effects were then studied through the trends of changes of a correction factor called spatial correction factor and its relative measurement uncertainty. The results from this study could be used to optimise the baffle condition and hence improving the sphere uniformity. It could consequently be further developed to be an economic and practical method for any existing sphere to improve the accuracy the measured total luminous flux, by the integrating sphere substitution method, of a linearly-shaped lamp.

\section{EXPERIMENTAL}

The integrating sphere used in this work was a 2-m-diameter UL2000 integrating sphere from LMT Lichtmesstechnik GMBH, at the centre of which a lamp was to be installed. The sphere inner wall was coated with LMT PHP80 paint with the percentage reflectance of $80 \% \pm 1.5 \%$. The right hemisphere was movable for sphere access. At the centre on the left hemisphere wall a photo-detector was placed, in front of which was a baffle. The detector was from LMT and had a field of view of $2 \pi$, with the spectral correction factor $f_{1}^{\prime}$ of $0.5 \%$ and the luminous responsivity of $18.96 \mathrm{nA} / \mathrm{lx}$. These characteristics, nevertheless, had no effect on the trend of changes of the measured total luminous flux value when the baffle changed, which was a main purpose of this study.

Figure 1 shows the schematic diagram of the sphere with the photo-detector and the baffle. Each position on the sphere inner wall was denoted angularly by two angles - vertical angle $\theta$ and horizontal angle $\phi$, where the reference position of $\theta=0$ was at the bottom of the sphere and $\phi=0$ at the hemisphere front-interface. Both angles were measured from their corresponding reference positions in a counter-clockwise direction as shown in Figure 1.

The experiment was started where the spatial response distribution function, $K$, of the sphere was determined. This parameter is the electronic signal obtained by the photodetector when a light source illuminates at a specific position of the sphere wall; hence it is a function of the sphere wall position, i.e. $K(\theta, \phi)$. The parameter was obtained by installing a sweeping LED at the centre of the sphere as shown in Figure 1. The sweeping LED set was from LMT Lichtmesstechnik GMBH and comprised of a LED and two stepping motors, namely $\theta$-motor and $\phi$-motor. The LED had an aperture of 33 $\mathrm{mm}$ diameter and emitted typical cool-white light, the spectrum of which can be found elsewhere [14]. Due to the aperture, the LED would directionally shine the light onto a specific angular position on the sphere wall. With the two stepping motors, the LED could be rotated to shine on every position on the sphere wall. To start the scanning procedure, firstly, the LED was arranged to illuminate at the beginning position, i.e. the bottom point of the sphere; $(\theta, \phi)=(0,0)$. The LED was biased at $3.5 \mathrm{~V}$ by using a power generator, providing a driven current of 0.17

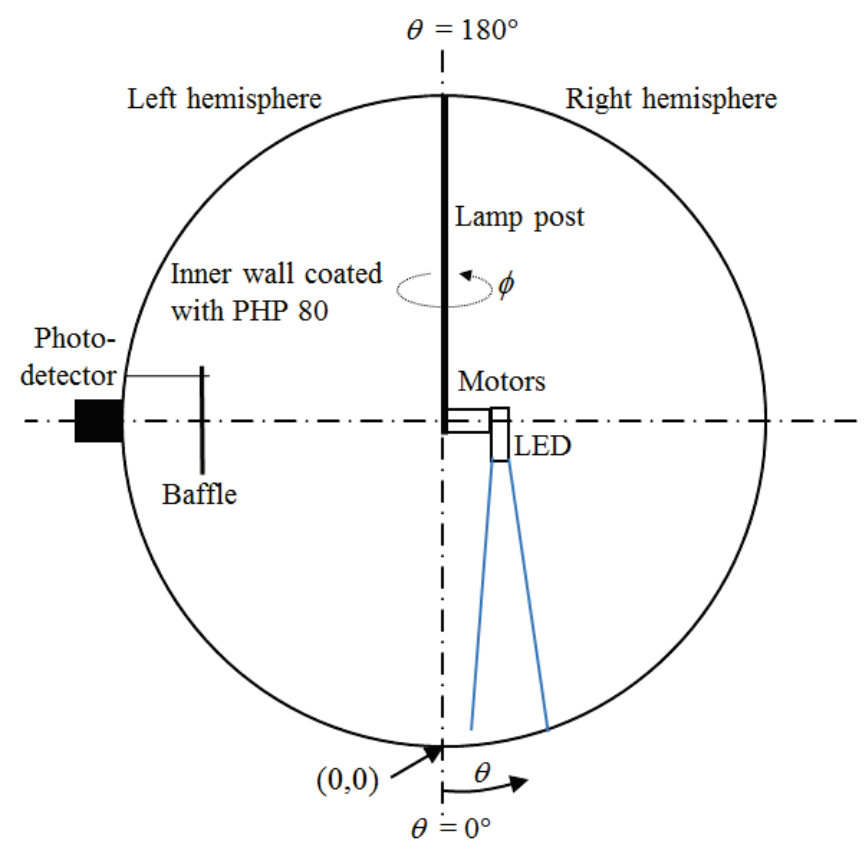

Figure 1. Front-viewed schematic diagram of the sphere with the sweeping LED set to obtain the spatial response distribution function, $K(\theta, \phi)$, at a specific angular position. 
A. Prior to the sweeping, the LED was left biased at this condition for two hours before the scanning was executed in order for the LED light to be stable. After two hours, the scanning started; the response signal $K(0,0)$, detected by the photo-detector at this beginning position, was collected by a computer program. Subsequently the $\theta$-motor rotated the angle $\theta$ by increasing at a $5^{\circ}$ interval, and thus the LED illuminated the new positions. At each position, the response signals, $K\left(5^{\circ}, 0\right), K\left(10^{\circ}, 0\right), K\left(15^{\circ}, 0\right)$, and so on, were collected, until the illuminated position was the top of the sphere and $K\left(180^{\circ}, 0\right)$ was collected. Then the $\theta$-motor rotated the LED back to $(0,0)$ while the $\phi$-motor rotated the angle $\phi$ for a step of $5^{\circ}$ around the vertical axis to be at $\left(0,5^{\circ}\right)$. With $\phi$ at $5^{\circ}$, the previous procedure was repeated where the LED was rotated horizontally; The response signals $K\left(0,5^{\circ}\right), K\left(5^{\circ}, 5^{\circ}\right), K\left(10^{\circ}, 5^{\circ}\right)$, and so on, until $K\left(180^{\circ}, 5^{\circ}\right)$, were collected. The LED was then rotated back to the position that $\theta=0$ and new $\phi$ increased by $5^{\circ}$. Such procedure was repeated until $\phi=360^{\circ}$, where $\left(180^{\circ}, 360^{\circ}\right)$ was the last illuminated position that the response signal was collected, i.e. the whole inner wall of sphere was swept and $K(\theta, \phi)$ for every angle $\theta$ and angle $\phi$ was obtained. This set of data was the $K$ profile of the sphere for a given sphere condition. For example, a different baffle would provide a different $K$ profile.

Apart from obtaining $K$ from the sphere, the luminous intensity distribution, $I$, of a standard spherical lamp and a linearly-shaped lamp were also acquired. This parameter is the luminous intensity, in the unit of candela, at each angular position around an illuminating lamp. Thus the parameter is a function of angular position on the sphere wall; i.e. $I(\theta, \phi)$.

The luminous intensity distribution at each angular position of the standard lamp, $I_{s}(\theta, \phi)$, and of the test lamp, $I_{t}(\theta, \phi)$, were obtained by using a mirror-type gonio-photometer from Photometric Solutions International, PSI. The operating principle of the gonio-photometer can be found elsewhere [15]. Briefly, each lamp was operated on the gonio-photometer while a mirror rotated around the lamp and reflected light to a photometer to collect its luminous intensity at a $5^{\circ}$ interval for both $\theta$ and $\phi$ angles, similarly to the steps of the $K(\theta, \phi)$. Once completed, the $I_{s}(\theta, \phi)$ and $I_{t}(\theta, \phi)$ profiles were obtained.

In this study, the standard lamp was a Polaron LF200 spherical incandescent lamp, and the test lamps were five linear fluorescent lamps, denoted as lamps $A, B, C, D$, and $E$. All test lamps had been preliminarily burnt for 300 hours prior to the experiment to ensure their emission stability during the experiment. All test lamps had different diameter, length, and colour correlated temperature (CCT) as shown in Table 1. The objectives of using such test lamps with different characteristics were to investigate whether the diameter, length, and CCT of the test lamps would affect the effect of baffle in the sphere. Figure 2 shows $I_{s}\left(90^{\circ}, \phi\right)$ and $I_{t}\left(90^{\circ}, \phi\right)$ profiles of the standard

Table 1. Details of the linearly-shaped test lamps.

\begin{tabular}{ccccc}
\hline Lamp & Diameter $(\mathbf{m m})$ & Length $(\mathbf{m m})$ & CCT $(\mathbf{K})$ & Color \\
\hline A & 26 & 1175 & 6120 & Daylight \\
B & 26 & 1175 & 2820 & Warm white \\
C & 26 & 560 & 6120 & Daylight \\
D & 26 & 560 & 3865 & Cool white \\
E & 16 & 1130 & 6120 & Daylight \\
\hline
\end{tabular}

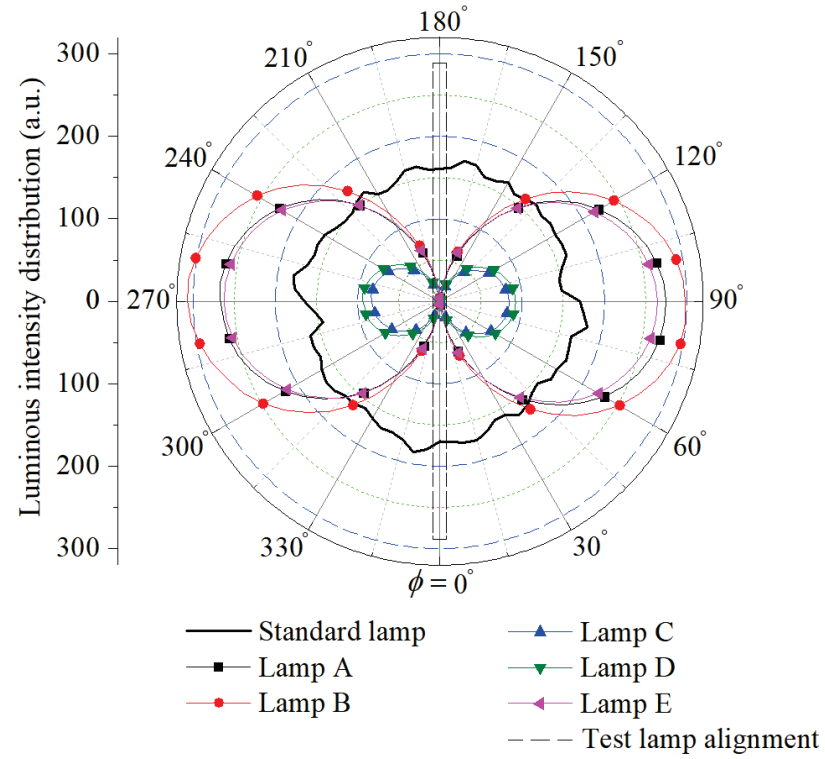

Figure 2. Luminous intensity distributions, $I_{s}\left(90^{\circ}, \phi\right)$ and $I_{t}\left(90^{\circ}, \phi\right)$, of the standard lamp and five linearly-shaped test lamps, respectively, at the plane $\theta=90^{\circ}$.

lamp and five linearly-shaped test lamps, respectively, at $\theta=$ $90^{\circ}$, which was the horizontally cross-sectional plane at the middle of the sphere. For the standard lamp, $I_{s}\left(90^{\circ}, \phi\right)$ looked roughly uniform for all angles $\phi$, corresponding to the spherical shape of the lamp. This was much different from those of linearly-shaped test lamps. For the test lamps, a pole of the lamp was set at the position $\phi=0$. For all test lamps, $I_{t}\left(90^{\circ}, 0\right)$ and $I_{t}\left(90^{\circ}, 180^{\circ}\right)$ were zero as they were at the poles of the lamp. The values gradually increased to maximum at $I_{t}\left(90^{\circ}, 90^{\circ}\right)$ and $I_{t}\left(90^{\circ}, 270^{\circ}\right)$ as such positions were at the middles of the lamp. The $I_{t}\left(90^{\circ}, \phi\right)$ of all lamps were of similar shape but with different magnitudes due to their different lengths; the lamps $A, B$, and $E$, provided expectably higher $I$ due to their longer lengths and hence higher power outputs.

$K(\theta, \phi), I_{s}(\theta, \phi)$, and $I_{t}(\theta, \phi)$ of each angular position $(\theta, \phi)$ were further used to calculate their normalised values, $K^{*}(\theta, \phi)$, $I_{s}^{*}(\theta, \phi)$, and $I_{t}^{*}(\theta, \phi)$, respectively, as follows [3].

$$
\begin{aligned}
& K^{*}(\theta, \phi)=\frac{4 \pi K(\theta, \phi)}{\int_{\phi=0}^{2 \pi} \int_{\theta=0}^{\pi} K(\theta, \phi) \sin \theta d \theta d \phi}, \\
& I_{s}^{*}(\theta, \phi)=\frac{I_{s}(\theta, \phi)}{\int_{\phi=0}^{2 \pi} \int_{\theta=0}^{\pi} I_{s}(\theta, \phi) \sin \theta d \theta d \phi}, \\
& I_{t}^{*}(\theta, \phi)=\frac{I_{t}(\theta, \phi)}{\int_{\phi=0}^{2 \pi} \int_{\theta=0}^{\pi} I_{t}(\theta, \phi) \sin \theta d \theta d \phi} .
\end{aligned}
$$

Subsequently, $K^{*}(\theta, \phi), I_{s}^{*}(\theta, \phi)$, and $I_{t}^{*}(\theta, \phi)$ were used to calculate the spatial correction factors of the standard lamp, $s c f_{s}$, and of the test lamp, $s c f_{t}$, as follows [3]:

$$
s c f_{s}=\frac{1}{\int_{\phi=0}^{2 \pi} \int_{\theta=0}^{\pi} I_{s}^{*}(\theta, \phi) K^{*}(\theta, \phi) \sin \theta d \theta d \phi},
$$




$$
s c f_{t}=\frac{1}{\int_{\phi=0}^{2 \pi} \int_{\theta=0}^{\pi} I_{t}^{*}(\theta, \phi) K^{*}(\theta, \phi) \sin \theta d \theta d \phi} .
$$

Consequently, the final spatial correction factor of the measuring system, $s c f$, was calculated as the ratio of $s c f_{t}$ and $s c f_{f}$ as follows:

$$
s c f=\frac{s c f_{t}}{s c f_{s}} .
$$

This $s c f$ is a factor indicating how different the measured total luminous flux value is from the accurate value when the luminous intensity distributions of the standard and test lamps are different.

Due to the normalised nature of both $I^{*}$ and $K^{*}$, the value of scf technically falls around one. The value of exactly one indicates that the measured total luminous flux is equal to the accurate value. If $s c f$ is less than one, the measured value is higher than the accurate value, and vice versa. However, $K$ can be affected by the scanning angular width and the aperture of the scanning LED [12], thus the absolute value of scf calculated in this study may deviate if those parameters are varied. As a purpose of this study is to investigate the trend of change of the measured total flux value, the attention was then focused only on the trend of scf regarding the change in the baffle conditions; i.e. if the change induced the increase in scf, the measured total luminous flux value was then decreased, and vice versa.

A source that causes the scf to deviate from one is the presence of the baffle. This is because the baffle creates two non-uniform characteristics on the sphere wall [12], [16]: (1) the baffle reflection, or the high-signal region, on the sphere wall around the detector behind the baffle, and (2) the baffle shadow, or the low-signal region, on the sphere wall opposite to the detector. In this report, these effects on the total luminous flux measurement of a linearly-shaped lamp were examined in three studies as follows:

a. Effects of the baffle reflection on the total luminous flux measurement of a linearly-shaped lamp with perpendicular alignment (i.e. the lamp was aligned perpendicularly to the line from the photo-detector as shown in Figure 3a).

b. Effects of baffle reflection and baffle shadow on the total luminous flux measurement of a linearly-shaped lamp with perpendicular alignment.

c. Effects of baffle reflection and baffle shadow on the total luminous flux measurement of a linearly-shaped lamp with coaxial alignment (i.e. the lamp was aligned along with the line from the photo-detector, as shown in Figure 3b).

The perpendicular and coaxial alignments were chosen for
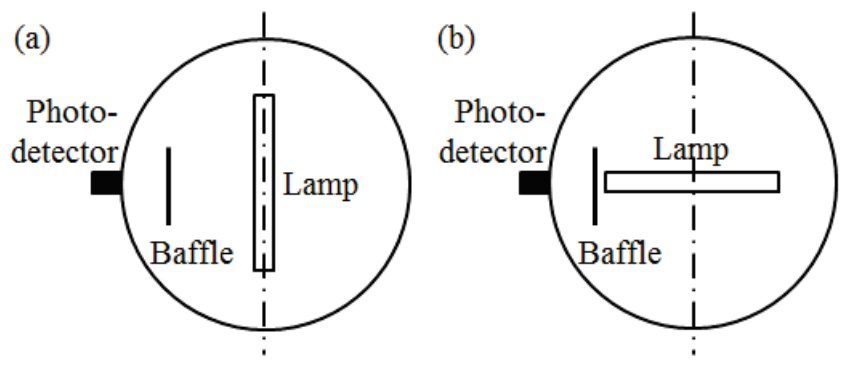

Figure 3. Top-viewed schematic diagram of a linearly-shaped test lamp in the sphere with (a) perpendicular and (b) coaxial alignments. study as they are the two common installing configurations of a linearly-shaped lamp inside an integrating sphere.

In each study, the effects of baffle reflection and baffle shadow were varied by changing the baffle size (in Studies a., b. and c.) and position (in Study a.). Then the corresponding scf was evaluated. In all studies, the baffles were painted with LMT PHP80 paint at both front and back sides. Thus their reflectances were controlled to be at $80 \% \pm 1.5 \%$, similar to that of the sphere wall, during the whole experiment.

The trend of change in the measurement uncertainty of scf was also examined. In each study, the measurement uncertainty of scf was calculated through the Monte-Carlo simulation [17][19]. This was processed by the factor being calculated repeatedly for 10,000 times. In each time, the parameters $K(\theta, \phi), I_{s}(\theta, \phi), I_{s}(\theta, \phi)$, and $\theta$, were randomly varied within their uncertainty ranges, and scf was calculated by using (1) - (6). Consequently, a total of 10,000 different scf values were obtained. Due to the random nature of the calculated parameters, the 10,000 scf values were normally distributed. However, with such a large number of $s c f$, its uncertainties estimated by using the normal distribution assumption were very small. Thus, in the following results, each uncertainty was estimated under the rectangular distribution to provide a larger value and to ensure that the uncertainty was covered in the range. This change in assumption had no effect on the trend of change in uncertainty, which was a purpose of this study.

Regarding the rectangular distribution of scf values, the absolute and relative uncertainties of $s c f$ were calculated by using the maximum $s c f, s c f_{\text {max }}$; minimum $s c f, s c f_{\text {min }}$; and mean $s c f, s c f_{\text {mean }}$, of the 10,000 scf values, as follows:

Absolute uncertainty of $s c f=\frac{s c f_{\max }-s c f_{\min }}{\sqrt{12}}$,

Relative uncertainty of $s c f=\frac{\text { Abs. unc. of } s c f}{s c f_{\text {mean }}} \times 100 \%$.

All the relative uncertainties reported in this study were with a coverage factor of $k=1$, corresponding to the level of confidence of $57.74 \%$ regarding the rectangular distribution [20].

\section{RESULTS AND DISCUSSIONS}

Figure 4 shows a typical normalised spatial response distribution function, $K^{*}$, of the left and right hemispheres of the sphere used in this experiment obtained by using a circular (a)

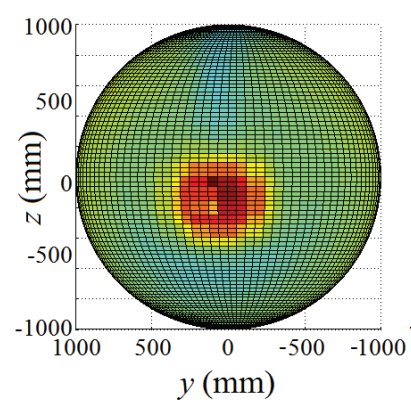

(b)

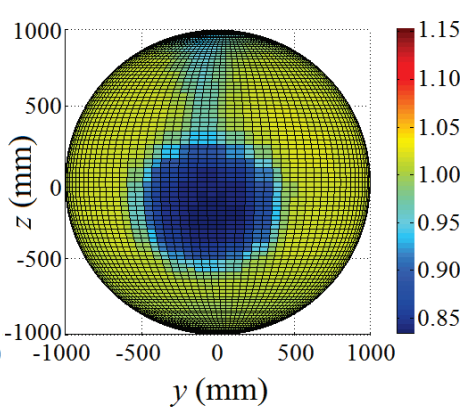

Figure 4. A 3-dimensional plot of a typical set of $K^{*}(\theta, \phi)$ of (a) left and (b) right hemispheres of the integrating sphere with a circular baffle; the horizontal axis, $y$, and the vertical axis, $z$, are the distances from the sphere centre. 
baffle. The baffle had a diameter of $120 \mathrm{~mm}$, or $12 \%$ of the sphere radius, and was located at $250 \mathrm{~mm}$, or $25 \%$ of the sphere radius, from the photo-detector.

The result indicated that $K^{*}$ was not uniform all over the sphere. Two distinct characteristics could be observed: (1) the high-signal region at the centre of the left hemisphere where the photo-detector and the baffle were located, and (2) the lowsignal region at the centre of the plain right hemisphere. Both anomalies are of circular shape as a result of the circular baffle.

Both characteristics were originated from the presence of the baffle [12]. The high-signal region at the left hemisphere was due to the baffle reflection; the light from the lamp was incident at the sphere wall around the baffle; it then incidented and reflected from the back surface of the baffle to the photodetector, hence the signal at that area higher than those in the surroundings. The low-signal region at the right hemisphere was due to the shadow of the baffle seen by the photo-detector. This is represented in Figure 5 which shows the relation between $K^{*}\left(90^{\circ}, \phi\right)$ and $\phi$, i.e. at the plane with $\theta=90^{\circ}$, the horizontally cross-sectional plane at the middle of the sphere. These two characteristics were expected to affect scf differently and were examined in the three following subsections.

\subsection{Effects of the baffle reflection on the total luminous flux measurement of a linearly-shaped lamp with perpendicular alignment}

The effect of the baffle reflection, i.e. the high-signal region, on scf was studied by the reflection being varied while the baffle shadow was controlled to have a constant size, and the corresponding scf was calculated. The experiment was carried out in a perpendicular alignment due to the space limitation in the sphere and the effect enhancement.

The baffle was in a racetrack shape as shown in Figure 6, with the width, $w$, fixed at $120 \mathrm{~mm}$, and the length, $l$, varied. To

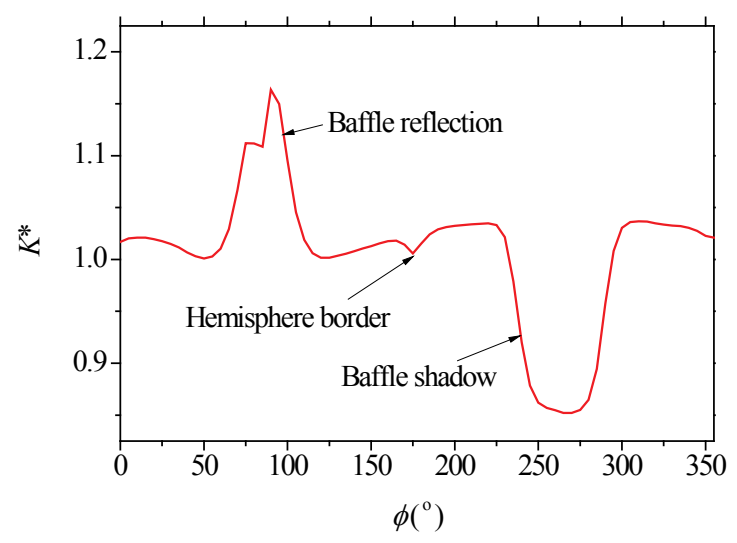

Figure 5. Relation between $K^{*}(\theta, \phi)$ and $\phi$, at $\theta=90^{\circ}$, i.e. the horizontally cross-sectional plane at the middle of the sphere.

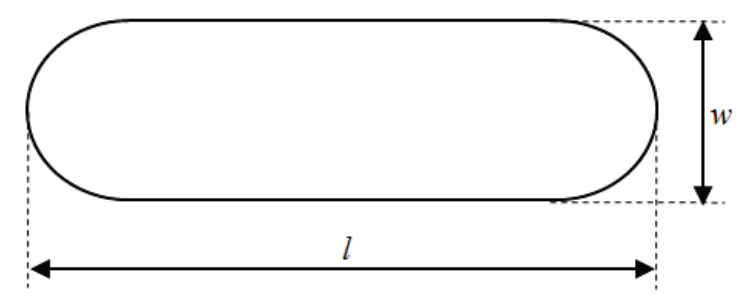

Figure 6 . Sketch of the racetrack shaped baffle with width $w$ and length $I$. vary the baffle reflection but not the baffle shadow, the baffle was positioned at different distances, $d$, from the photodetector. When $d$ increased, the baffle length, $l$, increased in the ratio that the baffle shadow on the opposite hemisphere at every baffle position was of the same size. The values $d$ of 106 $\mathrm{mm}, 154 \mathrm{~mm}, 202 \mathrm{~mm}$, and $250 \mathrm{~mm}$, therefore corresponded to the value $l$ of $220 \mathrm{~mm}, 320 \mathrm{~mm}, 420 \mathrm{~mm}$, and $520 \mathrm{~mm}$, respectively. At each position $d$, the $s c f$ was calculated. Figure 7 shows the schematic diagram of the sphere and baffle when the baffle was at the closest position to the photo-detector.

Figure 8 shows the effect of baffle distance, $d$, on scf and its corresponding relative uncertainty. The relations of all five test lamps are of similar trend and show that, once the baffle was further away from the photo-detector, scf increased, indicating the decrease in the measured total luminous flux value. As the baffle shadow (low-signal region) was the same for all baffle distances, the reason of this behaviour was mainly due to the baffle reflection (high-signal region) generated around the

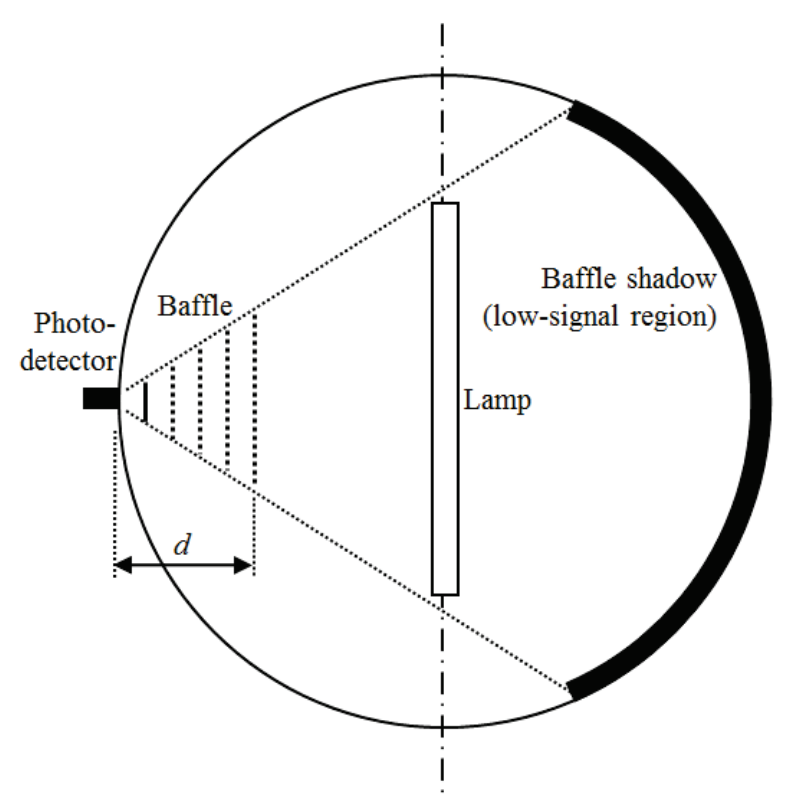

Figure 7. Top-viewed schematic diagram of the sphere with a perpendicular lamp alignment with the baffle position and size changed such that the baffle reflection varied but the baffle shadow remained the same. Solid line of the baffle shows its closest position to the photo-detector.

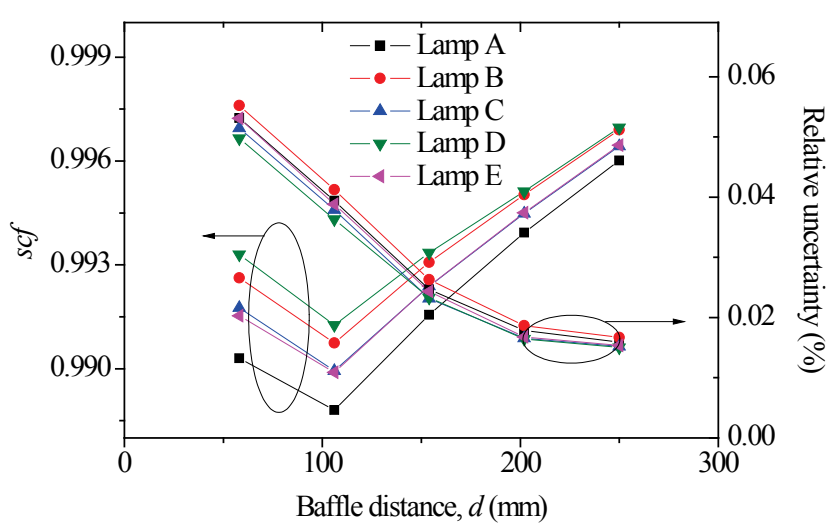

Figure 8. Relations between the baffle distance, $d$, and scf and its corresponding relative uncertainty, with a coverage factor of $k=1$, of linearly-shaped test lamps with perpendicular alignment. 
photo-detector. When the baffle became further away from the detector, light reflecting from the back of the baffle had decreasing magnitudes. This then led to the decrease in height of the signal at such area and the area size, resulting in the decreasing trend of the total luminous flux value.

The relative uncertainty of scf on this effect decreased with the increase in the baffle distance. This was expected to be a result from the fluctuation of the high signal in the reflection region. Once the baffle was further away from the detector, the signals were less fluctuated, leading to the narrower range of the signal and hence lower uncertainty.

\subsection{Effects of the baffle reflection and baffle shadow on the total luminous flux measurement of a linearly-shaped lamp with perpendicular alignment}

Here the baffle was in a racetrack shape with the width, $w$, fixed at $120 \mathrm{~mm}$, and the length, $l$, varied from $120 \mathrm{~mm}$ to 520 $\mathrm{mm}$. Its position $d$ was fixed at $250 \mathrm{~mm}$ from the photodetector. As the baffle position was fixed but its size changed, both the baffle reflection and shadow would be consequently changed simultaneously.

Figure 9 shows the relations between the length, $l$, of the baffle and scf of the system and its corresponding relative uncertainty. The relations of all test lamps exhibited a similar trend where scf decreased while its relative uncertainty increased with the increase in baffle length $l$. This indicates that, for this perpendicular arrangement, once the baffle became longer, the measured total luminous flux would increase.

This trend of scf was a combined effect of both baffle reflection and baffle shadow. Once the baffle length increased, both effects would be enhanced as both areas increased. The increases in baffle reflection and baffle shadow caused the increase and decrease, respectively, of the measured total luminous flux [12]. However, the increases in both effects were of different ratios where, in this lamp alignment, the effect of baffle reflection dominated. This was because once the baffle length increased, its edges became closer to the sphere wall around the photo-detector. This caused the magnitude of the high signal due to the baffle reflection to increase dramatically with the baffle length, while the magnitude of the low signal did not change. Consequently the effect of baffle reflection dominated that of the baffle shadow, resulting in the higher measured total luminous flux and the lower scf.

The relative uncertainty of $s c f$ in this lamp alignment was increased with the baffle length, indicating that, once the baffle

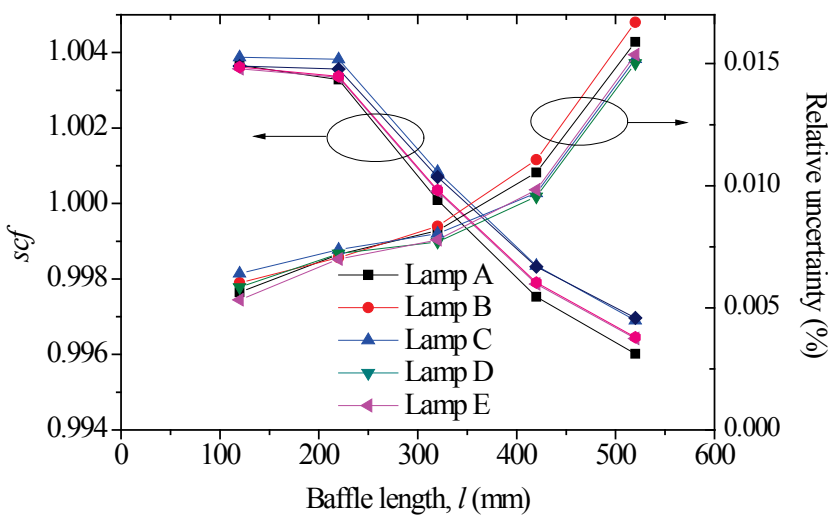

Figure 9. Relations between the baffle length, $l$, and $s c f$ and its corresponding relative uncertainty, with a coverage factor of $k=1$, of linearly-shaped test lamps with perpendicular alignment. length increased, the signal was more fluctuated. This was expected to be a result from the fluctuated signals in the baffle reflection region which became more fluctuated when the baffle was longer and closer to the sphere wall.

\subsection{Effects of the baffle reflection and baffle shadow on the total luminous flux measurement of a linearly-shaped lamp with coaxial alignment}

In this study, the lamp alignment was in a coaxial configuration as shown in Figure 3b, while the baffle was in a racetrack shape with width, $w$, of $120 \mathrm{~mm}$, and the varied length, $l$, from $120 \mathrm{~mm}$ to $520 \mathrm{~mm}$. In each $l$, scf was calculated.

Figure 10 shows the relations between the baffle length, l, and scf and its corresponding relative uncertainty. The relations show that the increase in baffle length caused scf increased, indicating the decreasing measured total luminous flux.

This is a combination effect of both baffle reflection and baffle shadow, where both increased with the increase in baffle length. However, the decrease in the measured total luminous flux value indicated that the increase in baffle shadow was more pronounced. This can be explained by using the areas of both regions as shown in Figure 11. When the baffle length

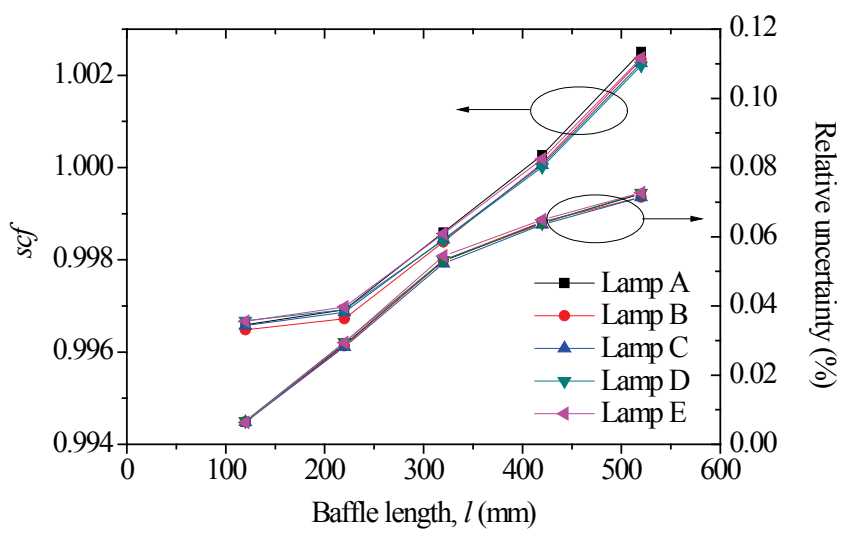

Figure 10. Relations between the baffle length, $l$, and $s c f$ and its corresponding relative uncertainty, with a coverage factor of $k=1$, of linearly-shaped test lamps with coaxial alignment.

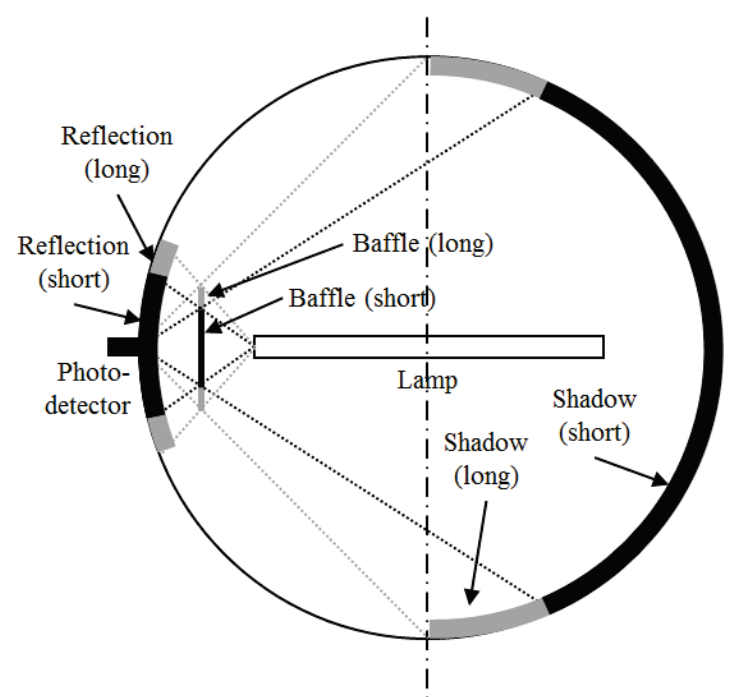

Figure 11. Schematic diagram of the sphere showing the areas of baffle reflection and baffle shadow when the baffle was short (black), and long (grey). 
increased, the areas of both shadow and reflection regions are both increased. However, as the shadow was further away from the photo-detector than the reflection, the size of the former increased at the higher ratio than the latter. Hence its effect dominated, resulting in the decrease in the measured total luminous flux value with the increase in baffle length.

Effects of the increase in area of baffle shadow caused the decrease in the measured total luminous flux value only in the coaxial alignment, not in the perpendicular alignment. This was because in the former, the increasing area of the baffle shadow was the area perpendicular to the middle of the lamp, which was the area with high luminous intensity distribution $I(\theta, \phi)$, while the reflection region was the area with low $I(\theta, \phi)$ from the lamp pole [21]. Thus, the shadow effect was more pronounced in the coaxial alignment. In the perpendicular alignment, the baffle reflection region was the area with high $I(\theta, \phi)$. Thus its effect was more pronounced as explained in the previous section.

The relative uncertainty of scf in this coaxial alignment increased with baffle length. This was because the increases in both baffle shadow and baffle reflection regions induced more fluctuated signals. It can be observed that the uncertainty of scf from this coaxial alignment was around 10 times higher than that in the perpendicular alignment obtained from the same baffle size. This indicated the poorer measurement performance of this alignment than that of the perpendicular one.

\section{CONCLUSIONS}

Baffle is a crucial component in an integrating sphere which is used in the measurement of the total luminous flux of a linearly-shaped light source. However, the presence of a baffle in front of the photo-detector in the sphere created two nonuniform characteristics of the sphere response on the sphere wall - (1) the baffle reflection, or the high signal region, around the detector behind the baffle, and (2) the baffle shadow, or the low signal region, on the opposite hemisphere. By using the spatial correction factor, these effects were found to differently cause the deviation of the value of measured total luminous flux from accurate. The increase in the baffle reflection caused the measured total luminous flux value to increase. On the contrary, the increase in the baffle shadow caused the value to decrease.

The increase in size of a baffle increased both effects. However, the dominant effect of the two depended on the lamp alignment. In the perpendicular lamp alignment, the effect of baffle reflection dominated that of the baffle shadow, resulting in the increase in the measured total luminous flux value, i.e. decrease in scf. This was because, when the baffle length increased, its edge was closer to the sphere wall and hence the height of the signal in the reflection region increased more dramatically compared to the baffle shadow. In the coaxial lamp alignment, on the contrary, the effect of baffle shadow dominated. This resulted in the decrease in the measured total luminous flux value, i.e. increase in $s c f$, when the baffle length increased. This was due to the increasing size of the shadow which was at a higher ratio than the increasing size of the reflection. Another reason was that the increasing shadow area was the area with high luminous intensity of the linearly-shaped lamp, and hence it was more effective than the increasing reflection area, which was the area with very low luminous intensity.

The increase in both baffle reflection and shadow also affected the uncertainty of the scf. Once the area of either region increased, the signals were more fluctuated, resulting in the increase in the measurement uncertainty. It was observed that the relative uncertainty of $s c f$ in the perpendicular alignment was 10 times lower than that in the coaxial alignment. This indicated that the former alignment could be used to improve the measurement uncertainty. Moreover, such alignment also provides more space for adjusting the baffle conditions to improve the measurement accuracy.

These trends on the measured total luminous flux value and its measurement relative uncertainty were similar regardless of the difference in the length and diameter of the linearly-shaped test lamps. This indicated that the results from this study could be applied to other linear shape lamps with different lengths and diameters from those used in the study.

As the presence of a baffle can affect the accuracy of the measured total luminous flux, its conditions including size and distance need to be carefully designed to provide the measured total luminous flux as accurately as possible. This paper focused mainly on the trend of change in the measured total fluxed value. Nevertheless, with further studies, the results could be developed thus that the baffle adjustment could be an economic and practical method to improve the accuracy the measured total luminous flux, by the integrating sphere substitution method, of a linearly-shaped lamp.

\section{ACKNOWLEDGEMENT}

KW would like to thank Rojana Leecharoen and Soontorn Chanyawadee, NIMT, for the help in sphere scanning programming; and Thayathip Thongtan, NIMT, and Siridech Boonsang, KMITL, for technical discussions.

\section{REFERENCES}

[1] C. DeCusatis, Handbook of Applied Photometry, Springer, 1997. ISBN 978-1-56396-416-9.

[2] Y. Ohno, New method for realizing a luminous flux scale using an integrating sphere with an external source, J. of the Illuminating Engineering Society, 24(1) (1995), pp. 106-115.

[3] Y. Ohno, Realization of NIST 1995 luminous flux scale using integrating sphere method, J. of the Illuminating Engineering Society, 25(1) (1996), pp. 13-22.

[4] F. Samedov, M. Durak, Realization of luminous flux unit lumen at National Metrology Institute of Turkey (UME), Optica Applicata, 34 (2004), pp. 265-274.

[5] M.-Q. Liu, X.-L. Zhou, W.-Y. Li, et al., Study on methodology of LED's luminous flux measurement with integrating sphere, J. of Physics D: Applied Physics, 41-14 (2008), pp. 144012.

[6] T. Poikonen, T. Pulli, A. Vaskuri, et al., Luminous efficacy measurement of solid-state lamps, Metrologia, 49 (2) (2012), pp. S135-S140.

[7] K. Ohkubo, M. Saito, K. Hirokawa, et al., Comparison of total luminous flux for integrating sphere photometers, J. of the Illuminating Engineering Institute of Japan (Shomei Gakkai Shi), 98(8A) (2014) pp. 362-368.

[8] T. Donsberg, T. Pulli, T. Poikonen, et al., New source and detector technology for the realisation of photometric units, Metrologia, 51(6) (2014), pp. S276-S281

[9] S. Park, D.-H. Lee, S.-N. Park, Six-port integrating sphere photometer with uniform spatial response, Applied Optics, 50(15) (2011) pp. 2220-2227.

[10] S. Park, D.-H. Lee, S.-N. Park, Experimental validation of the six-port design for a highly uniform integrating sphere photometer, Applied Optics, 52(29) (2013), pp. 7178-7185.

[11] K. Ohkubo, S. Mishima, Approximate cosine response detector head for integrating sphere photometers, J. of the Illuminating 
Engineering Institute of Japan (Shomei Gakkai Shi), 96(11) (2012) pp. 761-766.

[12] K. Wasapinyokul, R. Leecharoen, S. Chanyawadee, et al., Effects of integrating sphere conditions on the spatial response distribution function in the total luminous flux measurement, XX IMEKO World Congress, Busan, Republic of Korea, 2012.

[13] F. Lin, T. Li, D. Yin, et al., Research on effects of baffle position in an integrating sphere on the total luminous flux measurement, Proceedings of SPIE 9684 (2016), pp. 96841C.

[14] V. K, Khanna, Fundamentals of solid-state lighting: LEDs, OLEDs, and their applications in illumination and displays, CRC Press, New York. (2014), pp. 230.

[15] T. Yoshizawa, Handbook of optical metrology: Principles and applications, second edition, CRC Press, New York (2015), pp. 56.

[16] Y. Ohno, Detector-based luminous flux calibration using the absolute integrating-sphere method, Metrologia, 35 (1998), pp. 473-478.

[17] C. E. Papadopoulos, H. Yeung, Uncertainty estimation and
Monte Carlo simulation method, Flow Measurement and Instrumentation, 12 (2001), pp. 291-298.

[18] I. Farrance, R. Frenkel, Uncertainty in measurement: A review of Monte Carlo simulation using Microsoft excel for the calculation of uncertainties through functional relationships, including uncertainties in empirically derived constants, The Clinical Biochemist Reviews, 35(1) (2014), pp. 37-61.

[19] K. Godo, T. Saito, H. Shitomi, T. Zama, I. Saito, Development of a total luminous flux measurement facility for LEDs at the National Metrology Institute of Japan, 9th International Conference on New Developments and Applications in Optical Radiometry, Davos, Switzerland, (2005).

[20] Joint Committee for Guides in Metrology (JCGM), Evaluation of Measurement Data - Guide to the Expression of Uncertainty in Measurement, International Bureau of Weights and Measures (BIPM), (2008), pp. 70

[21] Y. Ohno and R. O. Daubach, Integrating sphere simulation on spatial nonuniformity errors in luminous flux measurement, J. of the Illuminating Engineering Society, 30(1) (2001), pp. 105-115. 